exhibited in the quaternionic notation as in the other. Moreover, we can write $\sigma . \Phi$ for $\sigma \cdot(\alpha \lambda+\beta \mu+\gamma \nu)$. This represents a vector which is a function of $\sigma$, viz. the function conjugate to $\boldsymbol{\Phi} . \sigma$; and $\boldsymbol{\sigma . \Phi .} . \rho$ may be regarded as the product of this vector and $\rho$. This is not so clearly indicated in the quaternionic notation, where it would be straining things a little to call $\mathrm{S} \sigma \phi$ a vector.

The combinations $\alpha \lambda, \beta \mu$, \&c., used above, are distributive with regard to each of the two vectors, and may be regarded as a kind of product. If we wish to express everything in terms of $i, j$, and $k, \Phi$ will appear as a sum of $i i, i j, i k, j i, j j, j k, k i, k j$, $k k$, each with a numerical coefficient. These nine coefficients may be arranged in a square, and constitute a matrix ; and the study of the properties of expressions like $\Phi$ is identical with the study of ternary matrices. This expression of the matrix as a sum of products (which may be extended to matrices of any order) affords a point of departure from which the properties of matrices may be deduced with the utmost facility. The ordinary matricular product is expressed by a dot, as $\Phi . \Psi$. Other important kinds of multiplication may be defined by the equa. tions-

$$
(\alpha \lambda) \underset{\times}{\times}(\beta \mu)=(\alpha \times \beta)(\lambda \times \mu), \quad(\alpha \lambda):(\beta \mu)=(\alpha . \beta)(\lambda . \mu) .
$$

With these definitions $\frac{1}{6} \Phi \times{ }_{\times}^{\times} \Phi: \Phi$ will be the determinant of $\Phi$, and $\Phi_{\times}^{\times} \Phi$ will be the conjugate of the reciprocal of $\Phi$ multiplied by twice the determinant. If $\Phi$ represents the manner in which vectors are affected by a strain, $\frac{1}{2} \Phi \times \Phi$ will represent the manner in which surfaces are affected, and $\frac{1}{6} \Phi_{\times}^{\times} \Phi: \Phi$ the manner in which volumes are affected. Considerations of this kind do not attach themselves so naturally to the notation $\phi=\alpha \mathrm{S} \lambda+\beta \mathrm{S} \mu$ $+\gamma \mathrm{S} \nu$, nor does the subject admit so free a development with this notation, principally because the symbol $\mathrm{S}$ refers to a special use of the matrix, and is very much in the way when we want to apply the matrix to other uses, or to subject it to various operations.

New Haven, Connecticut.

J. Williard GibBs.

\section{The Meaning of Algebraic Symbols in Applied Mathematics.}

Prof. Greenhill, on p. 462 (March i9), gives a naïve and most instructive description of the straits to which a "practical man" is put when he wishes to interpret the simplest general formula.

I have always held, not in sarcasm but in sorrow, that students brought up on the system of specialized and limited numerical formulæ used by Prof. Greenhill and some other Professors of Engineering in this country, must necessarily go through the tentative trial-and-error sort of process which he so graphically describes, whenever they have to obtain a numerical result from anything not already arithmetical. In other words they are unable to deal with complete algebraic symbols or concrete quantities.

The symbol " $v$ " to them does not completely represent a velocity, it only represents a number; and to make it represent a velocity some words, such as "in feet per second," must be added. Whereas, since it is plain that the velocity of light does not vary with its numerical specification, nor the size of a room change according as it is measured in feet or in inches, it is surely better to make a symbol express the essential and unchanging aspect of the thing to be dealt with, i.e. the thing itself, and not merely the number of some arbitrary and conventional units which the thing contains.

May I, then, assure Prof. Greenhill very seriously, and with cntire appreciation of and accord with his insistence that all expressions should be complete and capable of immediate practical numerical interpretation, that the equation $\mathrm{T}=\rho v^{2}$ is perfectly complete, and that it is true and immediately interpretable in every consistent system of units that has been or that can be invented ? $\mathrm{T}$ is the tenacity, $\rho$ the density, of the material of a ring, and $v$ is its critical or bursting velocity. There is no need to say a word more. And no properly taught student ought to have the slightest difficulty in obtaining a numerical result directly in any system of conventional units that may be offered him.

$$
\text { NO. I I I } 8 \text {, VOL. } 43]
$$

It is the frequent recurrence of such ghastly parodies of formulæ as-

$$
\mathrm{T}=\frac{62.4}{2240 \times 144} \cdot \frac{\rho v^{2}}{g}
$$

in many engineering treatises which makes them such dismal reading. It is a standing wonder to physicists how a man of Prof. Greenhill's power can fail to see the inadequacy and tediousness of expressions which are only true in one particular system of units, and which to be true even in that require the special statement of every unit employed. For not only is Prof. Greenhill's expression long in itself, but it is incomplete without the tiresome addition, " $\rho$ being measured in so and so, $v$ in \&c., $\mathrm{T}$ in something else, and $g$ meaning nothing more than the pure number $32 \cdot 2$." All this has been needlessly put into the formula, and so has to be wearisomely taken out again.

If there be any physicist who does not contend for the concrete interpretation of algebraic terms (wherein each symbol is taken to represent the quantity itself, and not merely a numerical specification of it in some conventional unit-see, for fuller explanation, NATURE, vol. xxxviii. p. 28I), I trust he will write and uphold his position on the side of Prof. Greenhill.

I suspect that the cause of Prof. Greenhill's failure at present to recognize the extreme simplicity and reasonableness of the physicist's procedure is to be found, partly in a vague idea that in order to get numerical results in British units from a general expression it is necessary to work it out in C.G.S. units first, and then translate, which I assure him is not the case; and partly in the general difficulty which most people feel in thinking it possible that they can be mistaken.

I would gladly convince Prof. Greenhill if I could, because he would carry with him so many other teachers, and thus a mass of waste labour would be saved annually to several thousands of students. Would it be too much to ask him to consider the matter with care, and, if possible, from our point of view ; setting me a sum to do if that would be any assistance towards bringing him to the desired point of view?

Oliver J. LODGE.

\section{Tension of a "Girdle of the Earth."}

IT is perfectly true, as Prof. Lodge has asserted, that a cord or chain running on its own track as an endless band in a friction. less groove of any form will not require the sides of the groove to keep it in that form. But whatever velocity it moves with, such a tension will exist all round it as to resist the centrifugal forces of its windings; and to preserve them by virtue of the curvature and constant tension, invariable in shape however the speed of coursing of the belt may be increased, without any ex. ternal guidance and assistance. If $w$ is the cord's mass per unit of its length, and $v$ the velocity with which it pursues its course, $z v v^{2}$ is the tension in dynes which will be set up all round it. The speed may of course be so increased as to tear the cord or chain to pieces; and this will occur in steel tires of railway wheels, for example, if the train's velocity on which the wheels are carried is much more than 120 miles an hour. Mr. Bourne long ago pointed out, in his works on the steam-engine, that a very low limit of speed in railway trains is enforced for safety in view of this dynamical condition so as not to approach and exceed working and proof-stresses at least in the material of which steel wheel-tires are formed.

But the truth of the proposition $\mathrm{T}=w v^{2}$ rests entirely on the supposition that the running cord or cable pursues exactly its own curve in its motion. For a tension of 30 tons per square inch to be reached in a maritime cable in virtue of its being carried round either at the equator or at any distance of latitude from the equator, it must be presupposed that while buoyed with its own submerged lightness so as to be practically weightless in the water, it must from one end point of support to the other follow accurately the equator's circle of curvature, or the circle of curvature of the small circle of latitude along which it is laid, because this is the line of motion along which its parts are carried along by the earth's rotation. These circles are practically straight lines for any mile or two of cable, and truly enough, if in the presence of even the weak force acting "centrifugally" on the cable's mass by the earth's rotation, it is attempted (supposing it to be quite weightless otherwise) to pull it as nearly straight as the hardly sensible curvature of the earth requires, mathematics would not yield its point an inch, and a pull of 